\title{
Photoelectron angular distributions from laser-excited aligned Yb atoms ionized by vacuum ultraviolet radiation
}

\author{
C Kerling, N Böwering and U Heinzmann \\ Fritz-Haber-Institut der MPG, D-1000 Berlin 33, Federal Republic of Germany \\ and \\ Fakultät für Physik, Universität Bielefeld, D-4800 Bielefeld 1, Federal Republic of Germany
}

Received 8 June 1990

\begin{abstract}
Using linearly polarized Ar I line radiation for ionization, the energy- and angle-resolved photoemission from $\mathrm{CW}$-laser-excited aligned $\mathrm{Yb}$ atoms in the $(6 \mathrm{~s} 6 \mathrm{p}){ }^{3} \mathrm{P}_{1}$ state is studied. Photoelectron angular distributions have been measured for different orientations of the laser polarization vector relative to the polarization vector of the ionizing radiation. From polynomial fit coefficients for these distributions the ratio of reduced dipole matrix elements and the phaseshift difference for the transitions $6 \mathrm{p} \rightarrow \varepsilon$ s and $6 \mathrm{p} \rightarrow \varepsilon d$ have been obtained at a photoelectron energy of $7.8 \mathrm{eV}$.
\end{abstract}

In order to approach a complete description of the atomic photoionization process and to gain experimental access to the relevant dipole transition matrix elements and phaseshift differences it is necessary to acquire information exceeding total cross section data. For single-photon ionization of atoms in isotropic initial states the angular distribution of the photoelectron emission is described by the second Legendre polynomial and only a single asymmetry parameter $\beta$ (Yang 1948). Further experimental information necessary for a more detailed description has to be gained from spin polarization measurements of the photoelectrons (Heinzmann 1980, Heckenkamp et al 1986, Svensson et al 1988) or from photoion alignment as measured from the polarization or angular distribution of the fluorescence radiation (Kronast et al 1986, Jiménez-Mier et al 1986) or via Auger electron angular distribution (Southworth et al 1983, Hausmann et al 1988). For photoionization of polarized initial states, however, the description of the photoelectron angular distribution is more complex involving associated Legendre polynomials of higher order and containing several independent parameters which yield additional-and in some cases complete-dynamical information without requiring spin polarization analysis. A general theoretical expression for photoelectron angular distributions from polarized atoms was given by Klar and Kleinpoppen (1982). Such experiments can be performed in a two-step process in which a polarized state is prepared via resonant laser excitation and subsequently ionized. Up to now, photoelectron angular distributions from laser-excited states have been investigated only in several atomic systems where visible or ultraviolet laser radiation could still be used for ionization (Hansen et al 1980, Chien et al 1983, Siegel et al 1983, Mullins et al 1985).

In this letter, we present photoelectron distributions from a selectively laser-excited state using -in contrast_vacuum ultraviolet (vUv) radiation for photoionization. In 
this way, the photon energy range can be extended to regions well above the ionization threshold. In the last decade, the combination of $\mathrm{CW}$ laser excitation and ionization by vuv synchrotron radiation for the study of various metal atoms has provided a wealth of detailed spectroscopic information on excited atomic states with respect to resonant photoionization and partial cross section (Bizau et al 1985, 1986, Preses et al 1985, Nunnemann et al 1985, Cubaynes et al 1989). In particular, Meyer et al (1987) varied the alignment of the excited state (by changing the laser polarization direction or by selecting a different fine-structure component of this state) to study and classify core-excited autoionizing states. However, information on both dipole matrix elements and phases could not be extracted from these measurements, since for intensity reasons these experiments were all performed with angle-integrated collection of the photoelectrons. Here, we describe angle-resolved studies of non-resonant photoemission from optically aligned target atoms using linearly polarized vUv line radiation from an intense discharge lamp. Likewise, intense synchrotron radiation could be used for similar experiments; measurements at distinct photon energies for resonant ionization by Zimmermann and co-workers (Zimmermann 1990) are in progress.

In our experiment, counterpropagating beams of linearly polarized laser and VUV radiation are used while fluorescence radiation and photoelectron emission is observed in a perpendicular direction. The set-up is represented schematically in figure 1. A boron nitride crucible is resistively heated to about $900 \mathrm{~K}$ to produce an effusive atomic beam of $\mathrm{Yb}$ with a density of about $10^{12}$ atoms $/ \mathrm{cm}^{3}$ in the interaction region. The $\mathrm{Yb}$ atoms in the $(6 s)^{2}{ }^{1} S_{0}$ ground state are excited to $(6 s 6 p){ }^{3} P_{1}$ by use of an actively stabilized single-mode ring dye laser at $\lambda=555.8 \mathrm{~nm}$ (typical output power: $300 \mathrm{~mW}$ ). The direction of the linear polarization of the laser (degree of polarization $P \approx 1.0$ ) can be rotated with a Fresnel rhomb. Although Doppler broadening results in an effective linewidth of the transition of about $850 \mathrm{MHz}$, the isotope and hyperfine structure of the transition (Schüler and Korsching 1938) was resolved. This enabled selective excitation of the isotopes ${ }^{174} \mathrm{Yb}$ which are most abundant $(\approx 32 \%)$ in natural ytterbium. The resonance transition was saturated and we expect the fraction of excited atoms to be close to the limit of $16 \%$. The fluorescence emitted from the excitation region centred $\approx 3.3 \mathrm{~mm}$ above the molybdenum nozzle of the furnace was imaged onto a photodiode using a lens and an aperture.

For photoionization, a capillary discharge lamp (Schönhense and Heinzmann 1983) was operated with argon producing vuv line radiation at photon energies of 11.63 and $11.83 \mathrm{eV}$ (Ar Ia and Ar Ib, respectively). In order to record the photoelectron angular distribution, the vUV radiation is linearly polarized by a rotatable three-mirror reflecting

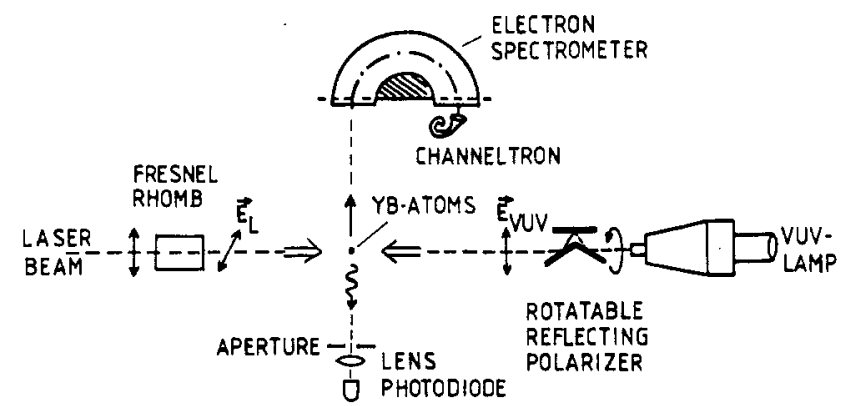

Figure 1. Experimental set-up for measurements of photoelectron angular distributions and excited-state fluorescence. 
polarizer (Hancock and Samson 1976) and the polarization vector is rotated relative to the detection direction of the electrons. With the method of Rabinovitch et al (1965), the degree of linear polarization was measured to be $P=0.91$ at typical intensities of some $10^{10}$ photons/s. Although the vUv lamp was operated with higher intensity at the helium resonance line the experiments reported here were carried out with argon $\mathrm{Ib}$ due to the much higher $6 \mathrm{p}$ photoionization cross section at this photon energy. Photoelectrons emitted perpendicularly to the photon beams in an electric- and magnetic-field-free region were energy-analysed with a simulated hemispherical spectrometer (Jost 1979) operated with a constant pass energy of $3.5 \mathrm{eV}$, at $100 \mathrm{meV}$ resolution and with $\pm 5^{\circ}$ angular acceptance and detected by a channeltron. Keeping the electron analyser fixed and rotating $\boldsymbol{E}_{\mathrm{Vuv}}$ has the advantage that the source volume viewed by the spectrometer does not change provided the vuv polarizer is adjusted sufficiently well to avoid any displacement or deviation of the outgoing beam during rotation. The adjustment was done with the help of a helium-neon laser.

In our experiment, the direction of the electric vectors $\boldsymbol{E}_{\mathrm{L}}$ and $\boldsymbol{E}_{\mathrm{VUV}}$ of both linearly polarised photon beams can be varied. The angle $\Theta$ between $\boldsymbol{E}_{\mathrm{vuv}}$ and the electron detection direction describes the photoelectron angular distribution. For completely linearly polarized light under our geometric conditions, the photoelectron angular distribution can be expressed in terms of associated Legendre polynomiais and five independent coefficients (Hansen et al 1980):

$$
I(\Theta)=\alpha_{00}+\alpha_{20} P_{20}(\cos \Theta)+\alpha_{40} P_{40}(\cos \Theta)+\alpha_{21} P_{21}(\cos \Theta)+\alpha_{41} P_{41}(\cos \Theta) \text {. }
$$

Thus, four coefficients $a_{i k}=\alpha_{i k} / \alpha_{00}$ can be determined from an angular distribution in relative measurements. These coefficients depend on the phase angle $\eta$ between the two electric vectors $\boldsymbol{E}_{\mathrm{L}}$ and $\boldsymbol{E}_{\mathrm{VUV}}$. Therefore the angular dependence is recorded preferentially by rotating both vectors simultaneously at constant phase angle. In general, the angular distribution depends not only on the transition matrix elements but also on the alignment of the laser-excited state as well as the degree of polarization of the light beams.

The transition ${ }^{1} \mathrm{~S}_{0} \rightarrow{ }^{3} \mathrm{P}_{1}$ for preparation of the excited ${ }^{174} \mathrm{Yb}$ atoms can in principle provide complete optical alignment, since only the $\left(m_{J}=0\right)$ sublevel of the excited state is populated with linearly polarized light. However, at the relatively high target densities needed for observation of photoelectrons from the laser-excited state a depolarization due to radiation trapping and collisions of excited atoms occurs (Fischer and Hertel 1982). The effective alignment $A$ was determined from the angular dependence of the resonance fluorescence at $555.8 \mathrm{~nm}$ measured with the photodiode while rotating the laser polarization direction (geometry $c$ of Fischer and Hertel 1982) $\dagger$.

In the experiments reported here we have examined the process:

$$
\begin{aligned}
\mathrm{Yb}^{*}(6 \mathrm{~s} 6 \mathrm{p}) & { }^{3} \mathrm{P}_{1}+h \nu_{\mathrm{vuv}} \\
& \rightarrow\left[\mathrm{Yb}^{+}(6 \mathrm{~s}){ }^{2} \mathrm{~S}_{1 / 2}+\mathrm{e}^{-}\left(\varepsilon \mathrm{s}_{1 / 2}, \varepsilon \mathrm{d}_{3 / 2}, \varepsilon \mathrm{d}_{5 / 2}\right)\right] \quad J=0,1,2 .
\end{aligned}
$$

The ionization potential for excited $\mathrm{Yb}^{3} \mathrm{P}_{1}$ is $4.02 \mathrm{eV}$ (Martin et al 1978). We have recorded photoelectron angular distributions at different phase angles with $\mathrm{Ar} \mathrm{Ib}$ radiation for photoelectrons with a kinetic energy of $7.81 \mathrm{eV}$. The distribution for parallel electric vectors has already been reported (Kerling et al 1989). Figure 2 shows

† We use the definition $A=a_{0}^{\mathrm{ph}} / F(F+1)$, where $a_{0}^{\mathrm{ph}}$ is the alignment parameter of Fischer and Hertel (1982) and $F$ denotes the total angular momentum including the nuclear spin. For $F=1$, the alignment $A$ is related to the state multipole moments $\rho_{i}$ according to: $A=\rho_{2} /\left(\sqrt{2} \rho_{0}\right)$. 

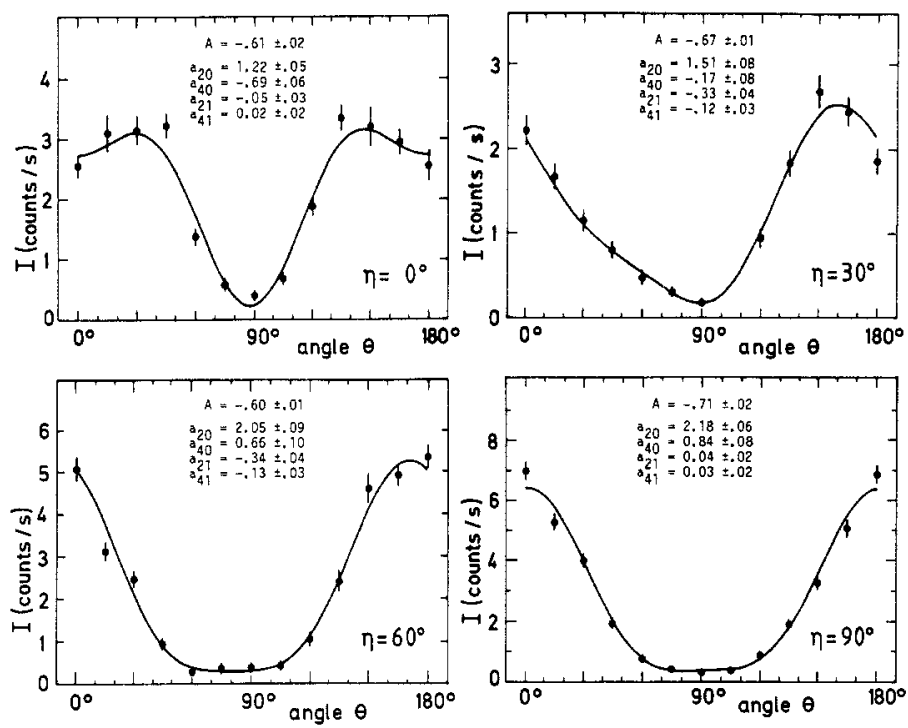

Figure 2. Angular distributions obtained at phase angles $\eta$ of $0,30,60$, and $90^{\circ}$. The data points are corrected for the background count rate (typically $0.5 \mathrm{~s}^{-1}$ ). The full curves originate from least-squares fits to the data. The corresponding polynomial fit coefficients and also the alignment $A$ (as obtained from fluorescence measurements) are given in the figures.

typical results obtained at four values of $\eta$ by measuring the photoelectron intensity at intervals of $15^{\circ}$ while rotating the polarization vectors of both laser and vuv beams synchronously. In each case, the corresponding effective target alignment was determined by recording the angular dependence of the fluorescence radiation simultaneously. Least-squares fits according to equation (1) to the data points yielded the polynomial coefficients $a_{i k}$ for each distribution.

When the electric vectors $\boldsymbol{E}_{\mathrm{L}}$ and $\boldsymbol{E}_{\mathrm{Vuv}}$ are parallel or perpendicular to each other the distributions are symmetric with respect to $\Theta=90^{\circ}$ (see also equation (3) below). In these cases the fit coefficients $a_{21}$ and $a_{41}$ are almost zero within the experimental error, as required. However, at other phase angles $\eta$ all four fit coefficients are distinctly different from zero and this symmetry disappears since now also odd functions involving terms containing $\sin \Theta \cos \Theta$ contribute. The shape of the angular distributions was found to be very sensitive to the degree of target alignment; distributions corresponding to higher alignment were more pronounced, because contributions from polynomials other than $P_{20}(\Theta)$ increase in relative strength. As $\eta$ is increased from $0^{\circ}$ to $90^{\circ} a_{20}$ becomes larger and $a_{40}$ changes its sign. At $\eta=90^{\circ} a_{20}$ and $a_{40}$ have equal sign and the spatial electron emission pattern is narrowest. Our angular distributions for a distinct angle $\eta$ resemble the corresponding distributions of Hansen et al (1980) and Siegel et al (1983) for $\eta+90^{\circ}$. The reason for this phaseshift of $90^{\circ}$ is that in our case of excited $\mathrm{Yb}^{3} \mathrm{P}_{1}$ the $6 \mathrm{p}$ electronic orbital is aligned perpendicular to the laser polarization vector while in their cases the excited electron orbital is aligned parallel to $E_{L}$.

As discussed by Klar and Kleinpoppen (1982), the dipole transition matrix elements and their relative phases can be extracted from the measured photoelectron angular distributions. We have applied their general formalism (equations (28-32) of Klar and Kleinpoppen 1982) to the state ${ }^{174} \mathrm{Yb}^{3} \mathrm{P}_{1}$, (nuclear spin $I=0$ ) for the case of the detection geometry of figure 1 and for ionization with linearly polarized light. As an 
extension, the equations were modified to include partially polarized vuv light. The angular distributions (see equation (1)) are then described in terms of the angles $\Theta$ and $\eta$, the alignment $A$ of the excited state, the degree of vuv polarization $P$, and several anisotropy coefficients $B$. In our case, six $B$ coefficients are relevant. They are functions of five transition matrix elements and four relative phases (cf equation (31) of Klar and Kleinpoppen 1982).

In order to simplify this rather complicated dependence and to obtain an approximate solution for the matrix elements as a first step, we have replaced these manyelectron matrix elements by non-relativistic single-electron matrix elements for the transition of the excited $6 \mathrm{p}$ electron to $\varepsilon \mathrm{s}$ and $\varepsilon \mathrm{d}$ continuum states. To that end the excited state was described in $j j$ coupling of the $6 \mathrm{p}$ electron with the core and it was assumed that the core angular momentum remains fixed during the ionization process. In addition, the spin-orbit interaction in the continuum was neglected, i.e. the spin of the electron was uncoupled. However, we take explicitly into account a singlet admixture to the excited state by expanding its wavefunction in terms of pure singlet and triplet state wavefunctions with normalized coefficients $\alpha$ and $\beta$ and introducing the abbreviation $\gamma=\alpha^{2}-2 \beta^{2}$. For $\mathrm{Yb}^{3} \mathrm{P}_{1}$, from the tables of Martin et al (1978) we find $\gamma=0.94$. The six relevant anisotropy coefficients then depend only on the transition amplitudes $D_{\mathrm{s}}$ and $D_{\mathrm{d}}$ of the reduced dipole matrix elements (outgoing $\varepsilon \mathrm{s}$ and $\varepsilon \mathrm{d}$ waves), their phaseshift difference $\Delta$ and the singlet-triplet mixture coefficient $\gamma$. Introducing the constant $C$ (which includes the population density of the laser-excited state), we then derive the following equations for the polynomial coefficients $\alpha_{i k}$ :

$$
\begin{aligned}
& \alpha_{00}=\frac{1}{24} C\left\{[8+2(1+3 P \cos 2 \eta) \gamma A] D_{\mathrm{s}}^{2}+(5-P)\left[2+\frac{1}{10}(5-3 \cos 2 \eta) \gamma A\right] D_{\mathrm{d}}^{2}\right. \\
& \left.-\sqrt{2}(1-P)[4+(1-3 \cos 2 \eta) \gamma A] D_{\mathrm{s}} D_{\mathrm{d}} \cos \Delta\right\} \\
& \alpha_{20}=\frac{1}{6} C\left\{\left[2 P+\frac{1}{14}(7 P+21 \cos 2 \eta)-18 P \cos 2 \eta \gamma A\right] D_{\mathrm{d}}^{2}\right. \\
& \left.-\sqrt{2}[4 P+(P+3 \cos 2 \eta) \gamma A] D_{\mathrm{s}} D_{\mathrm{d}} \cos \Delta\right\} \\
& \alpha_{21}=\frac{1}{4} C\left[\frac{1}{14}(7-3 P) \sin 2 \eta \gamma A D_{\mathrm{d}}^{2}-\sqrt{2} \sin 2 \eta \gamma A D_{\mathrm{s}} D_{\mathrm{d}} \cos \Delta\right] \\
& \alpha_{40}=\frac{18}{35} C P \cos 2 \eta \gamma A D_{\mathrm{d}}^{2} \\
& \alpha_{41}=\frac{9}{70} C P \sin 2 \eta \gamma A D_{\mathrm{d}}^{2} \text {. }
\end{aligned}
$$

For the special case $P=1$, these equations correspond to equation (3) of Hansen et al (1980) (if $\gamma A$ is replaced by their alignment parameter). From these equations and the experimental results for the normalized polynomial coefficients $a_{i k}$ and the corresponding alignment $A$, one can determine $\left(D_{\mathrm{s}} / D_{\mathrm{d}}\right)^{2}$ and the product $D_{\mathrm{s}} / D_{\mathrm{d}} \cos \Delta$; i.e. except for the sign, the ratio of the transition amplitudes $D_{\mathrm{s}} / D_{\mathrm{d}}$ and the cosine of the phaseshift difference $\Delta$ can be obtained. In principle, the data from a single angular distribution are sufficient for this analysis. We have therefore evaluated each distribution separately by forming the ratios $\alpha_{20} / \alpha_{40}$ and $\alpha_{40} / \alpha_{00}$ and solving for the two unknown quantities in these two equations. (For the data at $\eta=30^{\circ}$ and $60^{\circ}$, in addition, the ratio $\alpha_{21} / \alpha_{41}$ was used yielding consistent information.) The values determined agreed within the experimental errors. This proved the consistency of the data obtained at different degrees of alignment and different phase angles $\eta$ and also served to increase the accuracy of the values extracted. The average of the results from seven different distributions are:

$$
D_{\mathrm{s}} / D_{\mathrm{d}} \cos \Delta=-0.44 \pm 0.05 \quad \text { and } \quad\left(D_{\mathrm{s}} / D_{\mathrm{d}}\right)^{2}=0.28 \pm 0.07
$$


yielding

$$
|\cos \Delta|=0.83 \pm 0.14
$$

From equations (4) we can also derive an asymmetry parameter $\beta$ of $1.75 \pm 0.15$ for direct ionization of unpolarized ${ }^{174} \mathrm{Yb}(6 \mathrm{~s} 6 \mathrm{p}){ }^{3} \mathrm{P}_{1}$. Our results show that the amplitude of the $\varepsilon \mathrm{d}$ partial wave dominates; this is reasonable because photoionization occurs several $\mathrm{eV}$ above threshold. The results (4) do not yield complete information on the photoionization process, since at this point the corresponding absolute cross section and the sign of $\cos \Delta$ are not known. Our evaluation gives an approximate solution in which the non-relativistic treatment of the photoionization process is most likely the limiting step (see also Svensson et al 1988). Nevertheless, the results restrict the range of values for the matrix elements involved. Theoretical calculations are not available up to now for comparison with these experimental data.

The experiment described here represents a first step in vuv photoionization towards a complete analysis of the atomic ionization process in terms of transition amplitudes and phases from measurements of the photoelectron angular distribution alone. Although vUV radiation from a discharge lamp and not laser radiation is used for ionization, sufficiently high count rates can be achieved for non-resonant angle-resolved studies. Angular distribution coefficients and dipole matrix elements extracted from similar measurements in an extended vUV photon energy range will certainly be of interest for investigations of excited-state photoionization and future developments in related theoretical studies. In addition, our experiment opens the way to study electron correlations in VUV inner-shell ionization through the modification of the angular distribution due to laser excitation in the outer electron shell.

The authors are indebted to Professor A M Bradshaw for his encouragement, his generous support and the hospitality at the Fritz-Haber Institut. We would like to thank $\mathrm{H}$ Kerkhoff for his assistance with the computer fit program. Financial support by Deutsche Forschungsgemeinschaft (SfB 161, 216) and Bundesministerium für Forschung und Technologie (under contract 05331 AXI) is gratefully acknowledged.

\section{References}

Bizau J M, Cubaynes D, Gérard P, Wuilleumier F J, Picqué J L, Ederer D L, Carré B and Wendin G 1986 Phys. Rev. Lett. 57 306-9

Bizau J M, Wuilleumier F J, Ederer D L, Keller J C, Le Gouët J L, Picqué J L, Carré B and Koch P 1985 Phys. Rev. Lett. 55 1281-4

Chien R-L, Mullins O C and Berry R S 1983 Phys. Rev. A 28 2078-84

Cubaynes D, Bizau J M, Wuilleumier F J, Carré B and Gounaund F 1989 Phys. Rev. Lett. 63 2460-3

Fischer A and Hertel I V 1982 Z. Phys. A 304 103-17

Hancock W H and Samson J A R 1976 J. Electron Spectrosc. Relat, Phenom. 9 211-6

Hansen J C, Duncanson J A Jr, Chien R-L and Berry R S 1980 Phys. Rev. A 21 222-33

Hausmann A, Kämmerling B, Kossmann H and Schmidt V 1988 Phys. Rev. Lett. 61 2669-72

Heckenkamp Ch, Schäfers F, Schönhense G and Heinzmann U 1986 Z. Phys. D 2 257-74

Heinzmann U 1980 J. Phys. B: At. Mol. Phys. 13 4353-66, 4367-81

Jiménez-Mier J, Caldwell C D and Ederer D L 1986 Phys. Rev. Lett. 57 2260-3

Jost K 1979 J. Phys. E: Sci. Instrum. 12 1001-5, 1006-12

Kerling C, Böwering N and Heinzmann U 1989 Proc. 16th Int. Conf. on the Physics of Electronic and Atomic Collisions (New York) ed A Dalgarno, R S Freund, M S Lubell and T B Lucatorto (Amsterdam: North-Holland) Abstracts p 9

Klar H and Kleinpoppen H 1982 J. Phys. B: At. Mol. Phys. 15 933-50 
Kronast W, Huster R and Mehlhorn W 1986 Z. Phys. D 2 285-96

Martin W C, Zalubas R and Hagan L 1978 Atomic Energy Levels-The Rare-Earth Elements NSRDS-NBS 60 (Washington, DC: US Govt Printing Office) p 378

Meyer M, Müller B, Nunnemann A, Prescher Th, von Raven M, Schmidt M, Sonntag B and Zimmermann P 1987 Phys. Rev. Lett. 59 2963-6

Mullins O C, Chien R-L, Hunter J E III, Keller J S and Berry R S 1985 Phys. Rev. A 31 321-8

Nunnemann A, Prescher Th, Richter M, Schmidt M, Sonntag B, Wetzel H E and Zimmermann P 1985 J. Phys. B: At. Mol. Phys. $18337-41$

Preses J M, Burkhardt C E, Corey R L, Earsom D L, Daulton T L, Garver W P, Leventhal J J, Msezane A Z and Manson S T 1985 Phys. Rev. A 32 1264-6

Rabinovitch K, Canfield L R and Madden R P 1965 Appl. Opt. 4 1005-10

Schönhense G and Heinzmann U 1983 J. Phys. E: Sci. Instrum. 16 74-82

Schüler H and Korsching H 1938 Z. Phys. $111386-90$

Siegel A, Ganz J, Bußert W and Hotop H 1983 J. Phys. B: At. Mol. Phys. 16 2945-59

Southworth S, Becker U, Truesdale C M, Kobrin P H, Lindle D W, Owaki S and Shirley D 1983 Phys. Rev. A $28261-79$

Svensson A, Müller M, Böwering N, Heinzmann U, Radojevic V and Wijesundera W $1988 \mathrm{~J}$. Phys. B: At. Mol. Opt. Phys. 21 179-85

Yang C N 1948 Phys. Rev. 74 764-72

Zimmermann P 1990 Invited Talk DPG meeting München 